\title{
Prevalência de hábitos de sucção não nutritivos em pré-escolares e a percepção dos pais sobre sua relação com maloclusões
}

\author{
Prevalence of non-nutritive sucking habits in preschoolers \\ and parents' perception of its relationship with malocclusions
}

Cléa Adas Saliba Garbin ${ }^{1}$

Artênio José Ísper Garbin ${ }^{1}$

Ronald Jefferson Martins ${ }^{1}$

Neila Paula de Souza ${ }^{1}$

Suzely Adas Saliba Moimaz ${ }^{1}$
${ }^{1}$ Departamento de Odontologia Infantil e Social, Faculdade de Odontologia de Araçatuba, Universidade Estadual Paulista. R. José Bonifácio 1193, Vila Mendonça. 16.015-050 Araçatuba SP Brasil.rojema@foa.unesp.br
Abstract This cross-sectional and descriptive study sought to verify the prevalence of sucking habits in preschoolers and their parents' perception of its relationship with the occurrence of malocclusion. The population consisted of a representative sample of parents of preschoolers aged between 4 months and 6 years old. A semi-structured, self-administered questionnaire was used, consisting of open and closed questions relating to frequency of use and knowledge of parents regarding non-nutritive sucking habits. Of the 356 respondents, $70.8 \%$ stated that the children had some oral habit, with pacifier sucking being the most frequent (45.6\%). Although the majority (97.1\%) of the parents or caregivers admitted being aware that these habits could be detrimental to teeth, $70.2 \%$ of the respondents had already offered a pacifier to children, in most cases to calm them (61.8\%). There was a statistically significant association between offering a pacifier to a child and knowledge about the relationship of the existence of non-nutritive habits and the occurrence of malocclusion ( $p<0.0001$ and Chi-square $=60.123$ ). The conclusion is that the prevalence of oral habits of the population is high and, despite the majority of parents knowing that pacifier sucking can cause damage to oral health, they offer them to calm the child.

Key words Sucking behavior, Pacifiers, Preschooler
Resumo Este estudo transversal e descritivo objetivou verificar a prevalência de hábitos de sucção em pré-escolares e a percepção dos pais sobre a relação com a ocorrência de maloclusões. A população do estudo constituiu-se por uma amostra representativa de pais de pré-escolares de 4 meses a 6 anos de idade. Utilizou-se um questionário semiestruturado, composto por questões abertas e fechadas, referentes à frequência e conhecimentos dos pais frente aos hábitos de sucção não nutritivos. Dos 356 participantes da pesquisa, 70,8\% afirmaram que as crianças apresentavam algum hábito bucal, sendo a sucção de chupeta o mais frequente (45,6\%). Apesar da grande maioria dos pesquisados $(97,1 \%)$ relatarem saber que os hábitos podiam causar prejuizo aos dentes, 70,2\% deles já haviam oferecido chupeta à criança, na maioria das vezes para acalmá-la (61,8\%). Houve associação estatisticamente significativa entre a oferta da chupeta à criança e o conhecimento sobre a relação da presença de hábitos não nutritivos e a ocorrência de maloclusão ( $p<0,0001 e$ Qui-quadrado $=60,123)$. A prevalência de hábitos bucais na população estudada é alta e, apesar da maioria dos pais saberem que o hábito de sucção de chupeta pode causar danos à saúde bucal, ofertavam a chupeta a fim de acalmar a criança. Palavras-chave Comportamento de sucção, Chupetas, Pré-escolar 


\section{Introdução}

Desde a vida intrauterina, o bebê apresenta o reflexo natural de sucção não nutritiva, por meio das mãos e dedos ${ }^{1}$. Essas ações podem ser visualizadas em registros ultra-sonográficos fetais a partir da vigésima semana de gestação ${ }^{2}$, sendo que ao nascer o bebê apresenta a função de sucção completamente desenvolvida ${ }^{3}$.

A sucção não nutritiva proporciona à criança sensações de bem-estar, prazer emocional, proteção $0^{4}$, conforto ${ }^{5}$ e satisfação, suprindo suas carências afetivas e psicológicas ${ }^{6}$, podendo vir a se tornar um hábito após o nascimento, pois muitas vezes a criança atinge a sensação de plenitude alimentar, mas não supre suas necessidades emocionais ${ }^{7}$. Em especial, a sucção de chupeta destaca-se pela alta prevalência ${ }^{8,9}$, sendo um dispositivo amplamente utilizado por crianças em todo o mundo e que apresenta forte caráter cultural ${ }^{10,11}$.

O hábito pode provocar maloclusões ${ }^{6,12-14}$, sendo que a resistência dento-alveolar, o padrão dento-facial da criança e os fatores ligados ao próprio hábito de sucção (Tríade de Graber) vão determinar a ocorrência, o tipo e a gravidade das alterações oclusais, faciais e musculares. A Tríade é constituída pela intensidade, que é a força aplicada durante o hábito; pela frequência, que corresponde ao número de vezes que o hábito é executado; e pela duração, que é o período de tempo na qual o hábito é praticado ${ }^{15}$.

A relação entre hábitos de sucção não nutritivos e anormalidades dento-faciais é relatada por vários autores ${ }^{6,12-14}$; entretanto, são raros os estudos que procuraram verificar a percepção dos pais/responsáveis das crianças sobre essa relação. Nesse contexto e partindo do pressuposto de que o conhecimento dos pais pode interferir categoricamente na frequência e na duração da prática dos hábitos não nutritivos de seus filhos, objetivou-se verificar a prevalência de hábitos de sucção em pré-escolares e a percepção dos pais sobre a relação com a ocorrência de maloclusões.

\section{Metodologia}

\section{Tipo de estudo}

Trata-se de um estudo epidemiológico, transversal e descritivo; aprovado pelo Comitê de Ética em Pesquisa com Seres Humanos (CEP) da Faculdade de Odontologia de Araçatuba (UNESP).

No início, o pesquisador responsável pela coleta de dados dirigiu-se às escolas com o obje- tivo de explicitar a finalidade da pesquisa, os procedimentos adotados e o posterior uso dos dados coletados, a fim de obter as autorizações dos diretores para realizá-la.

\section{Amostragem}

Conforme dados fornecidos pela Secretaria Municipal de Educação, no período do estudo existiam 4325 pré-escolares com idades de 4 meses a 6 anos de idade, de ambos os sexos, regularmente matriculados nas 36 Escolas Municipais de Educação Básica (EMEB) do município de Araçatuba (SP).

Dividiu-se o mapa da cidade em cinco setores, sendo um central e quatro periféricos com o propósito de obter uma amostra representativa de pais ou responsáveis de crianças com diferentes características socioeconômicas. Selecionouse por sorteio uma escola em cada setor da cidade. Nas três regióes que possuíam populações maiores foram sorteadas mais uma escola em cada, no final totalizando oito escolas.

O dimensionamento da amostra foi calculado utilizando-se o método probabilístico, considerando-se a população finita, variáveis nominais e nível de confiança de $5 \%{ }^{16}$. O número estimado inicialmente para compor a amostra foi de 353 pais ou responsáveis de pré-escolares matriculados em uma das oito escolas.

Devido a possíveis perdas foram aplicados 424 questionários, o que corresponde a um acréscimo de $20 \%$ do total inicialmente estimado. Em cada escola, o tamanho da amostra foi obtido por meio do método da Probabilidade Proporcional ao Tamanho (PPT). Esse método permite que a proporcionalidade existente entre as escolas seja respeitada.

Os pais/responsáveis pelas crianças com idade superior a 6 anos, ou portadoras de fendas lábio-palatinas foram excluídos da amostra. Também não participaram do estudo os responsáveis que não assinaram o Termo de Consentimento Livre e Esclarecido (TCLE).

\section{Método de Coleta de Dados}

A coleta dos dados foi realizada através de entrevistas, nos dias de reuniões de pais e mestres, com perguntas estruturadas e semiestruturadas sobre a presença de hábitos bucais não nutritivos nas crianças e o conhecimento dos pais sobre a relação desses hábitos com a ocorrência de maloclusões. Realizou-se pré-teste com o objetivo de adequar o questionário antes de sua 
Os dados coletados foram tabulados por meio do programa Bioestat, versão 5.0 ${ }^{17}$, analisados estatisticamente por meio dos Testes Quiquadrado e Exato de Fischer, com nível de significância de $5 \%$ e apresentados em frequências absolutas e percentuais. Utilizou-se o método da Análise de Conteúdo proposta por Bardin ${ }^{18}$ para a análise das questões abertas.

\section{Resultados e discussão}

No Brasil, segundo o último levantamento epidemiológico realizado em nível nacional, a maloclusão figura-se entre os principais problemas de saúde bucal ${ }^{19}$. A sua relação com hábitos de sucção não nutritivos já foi estabelecida em outros estudos ${ }^{13,14,20}$.

Neste estudo, a taxa de resposta foi de 355 $(83,7 \%)$ questionários, número acima do estimado inicialmente para compor uma amostra representativa de país/responsáveis de pré-escolares do município estudado. Dentre os participantes da pesquisa, 179 (50,4\%) eram do sexo masculino.

Verificou-se que a maioria das crianças apresentava um ou mais hábitos bucais deletérios 248 $(69,9 \%)$, sendo a sucção de chupeta o mais fre-

quente 252 (44,8\%), seguido pelos hábitos de roncar, chupar os dedos e ranger os dentes; concordando com outros estudos onde esse hábito também se mostrou como o de maior prevalência ${ }^{9,21}$ (Tabela 1).

A chupeta é um dispositivo de preço reduzido, acessível à maioria da população $\mathrm{o}^{22} \mathrm{e}$ que possui uma associação simbólica com a figura do recém-nascido. Tradicionalmente apresenta-se como um dos itens do enxoval do bebê $\hat{e}^{11}$, constatação observada no presente estudo, onde em aproximadamente $50 \%$ dos enxovais das crianças a chupeta estava presente.

Em relação à oferta deste artefato à criança, $233(65,6 \%)$ pais ou responsáveis relataram que já haviam oferecido chupeta à criança (Tabela 2); entretanto, cabe ressaltar que nem todas as crianças para as quais a chupeta é oferecida desenvolvem o hábito de sucção. É de conhecimento popular que as crianças que usam chupeta são mais calmas, choram menos e dormem mais facilmente, servindo esse artefato como calmante e consolo para o bebêt ${ }^{11}$. Essas evidências corroboram os resultados deste estudo, onde 131 (56,2\%) pais ou responsáveis pelas crianças afirmaram que o principal motivo da oferta da chupeta era para acalmar a criança ou fazê-la parar de chorar. Quanto à frequência de uso, 47 (42,7\%) crianças usavam-na por mais de 6 horas diárias. Evidências clínicas e experimentais sugerem que na criança que realiza a sucção por um período de quatro a seis horas diárias, haverá uma movimentação dental significativa ${ }^{23,24}$.

Tabela 1. Distribuição numérica e percentual das crianças, de acordo com o tipo de hábito deletério, assim como o período realizado, Araçatuba (SP), 2012.

\begin{tabular}{|c|c|c|c|c|c|c|c|c|c|c|c|}
\hline \multirow[b]{2}{*}{ Hábitos } & \multicolumn{4}{|c|}{ Duração em anos } & \multicolumn{7}{|c|}{ Período } \\
\hline & $\mathbf{n}$ & $\begin{array}{l}\text { Até } 3 \\
\text { anos }\end{array}$ & $\begin{array}{c}\text { Acima } \\
\text { de } 3 \\
\text { anos }\end{array}$ & $\begin{array}{c}\text { Não } \\
\text { respondeu }\end{array}$ & Dia & Noite & $\begin{array}{c}\text { Somente } \\
\text { para } \\
\text { dormir }\end{array}$ & $\begin{array}{l}\text { Dia e } \\
\text { noite }\end{array}$ & $\begin{array}{c}\text { Somente } \\
\text { na } \\
\text { escola }\end{array}$ & Raramente & $\begin{array}{l}\text { Não } \\
\text { respondeu }\end{array}$ \\
\hline Chupar chupeta & 113 & 57 & 56 & 0 & 3 & 17 & 28 & 54 & 0 & 2 & 6 \\
\hline Chupar os dedos & 29 & 7 & 22 & 1 & 2 & 4 & 7 & 15 & 0 & 0 & 1 \\
\hline Roncar & 58 & 16 & 42 & 0 & 0 & 19 & 21 & 10 & 0 & 6 & 2 \\
\hline Babar & 15 & 0 & 15 & 0 & 0 & 7 & 2 & 4 & 0 & 2 & 0 \\
\hline Ranger os dentes & 29 & 9 & 16 & 4 & 1 & 14 & 3 & 2 & 0 & 5 & 4 \\
\hline Roer as unhas & 20 & 4 & 11 & 0 & 4 & 0 & 3 & 1 & 0 & 8 & 4 \\
\hline Respirar pela boca & 25 & 13 & 12 & 0 & 0 & 0 & 7 & 2 & 0 & 9 & 7 \\
\hline Outros & 5 & 2 & 3 & 0 & 0 & 0 & 4 & 0 & 0 & 1 & 0 \\
\hline Nenhum hábito & 103 & 23 & 73 & 7 & 0 & 0 & 0 & 0 & 0 & 0 & 0 \\
\hline Não respondeu & 4 & 0 & 0 & 0 & 0 & 0 & 0 & 0 & 0 & 0 & 0 \\
\hline
\end{tabular}

Obs.: algumas crianças apresentavam mais de um hábito. 


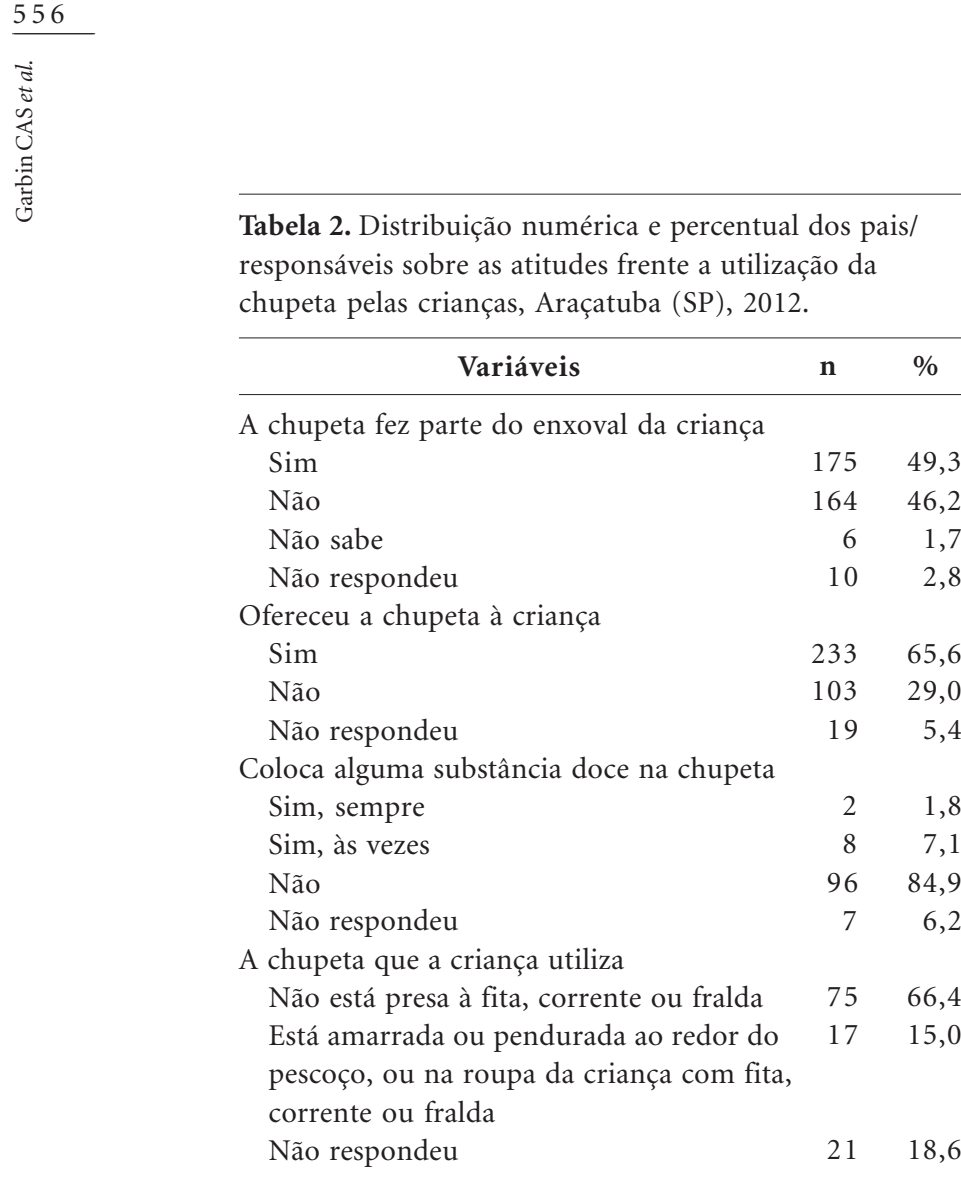

A prática de amarrar ou pendurar a chupeta ao redor do pescoço, ou na roupa da criança com fita, corrente ou fralda foi relatada por 17 (15\%) pais ou responsáveis. Essa atitude não deve ser adotada pelos pais, pois permite que a chupeta esteja sempre à disposição da criança reforçando o hábito, além de haver a possibilidade de acidentes, como o estrangulamento da mesma ${ }^{25}$. Obrigatoriamente, todas as embalagens desses dispositivos devem conter a recomendação de não colocar laços ou fitas para prender a chupeta ao pescoço, de acordo com a Norma Brasileira (NBR) 10334 do Instituto Nacional de Metrologia, Qualidade e Tecnologia (INMETRO) ${ }^{26}$.

Quanto à percepção dos pais/responsáveis pelas crianças sobre a relação dos hábitos de sucção não nutritivos com a ocorrência de maloclusões, a grande maioria, 303 pais $(97,1 \%)$, acreditava que os hábitos de sucção não nutritiva poderiam causar prejuízo aos dentes das crianças, sendo que desses 150 (42,25\%) relataram que conheciam os possíveis danos. Os mais citados foram dentes tortos 87 (58\%), problemas na fala 10 $(6,7 \%)$, má formação da arcada dentária $9(6 \%)$, palato profundo $7(4,6 \%)$, entre outros. A cárie dentária também foi mencionada, mas essa do- ença infecto-contagiosa não é causada diretamente pelos hábitos; o risco dessa doença poderá ser aumentado quando acrescenta-se algum tipo de substância doce à chupeta da criança. Verificouse no presente estudo que a maior parte dos pais das crianças 96 (84,5\%) afirmou que não realizavam essa ação, corroborando a recomendação da American Dental Association (ADA) ${ }^{27}$.

Quanto à tentativa de remoção do hábito de sucção, 84 (33,3\%) pais/responsáveis das crianças que apresentavam algum hábito deletério, relataram que já haviam efetuado essa ação. A conversa com a criança solicitando que ela abandonasse o hábito de sucção de dedo ou chupeta devido à idade ou explicando a possibilidade da ocorrência de problemas bucais foi o método empregado com maior frequência 54 (21,4\%); seguido pela oferta de presente ou recompensa em troca do abandono do hábito 17 (6,7\%), a procura pelo dentista para instalação de aparelho que impedia a realização do hábito 8 (3,2\%); o uso de pimenta ou substância amarga na chupeta ou dedo da criança $8(3,2 \%)$; além de outras formas de tentativa de remoção do hábito 8 (3,2\%). Em 39 (15,5\%) dos casos as crianças abandonaram o hábito por si mesmas ou por sentirem vergonha dos amigos. Normalmente, há a diminuição da frequência dos hábitos com aumento da idade; entretanto, caso a criança não pare por iniciativa própria, os pais devem desestimulá-la entre o terceiro e o quarto anos de vida ${ }^{20,27}$. No geral, as ameaças ou medidas punitivas não apresentam resultado positivo na tentativa de motivação da criança em deixar o hábito ${ }^{23}$.

No presente trabalho, verificou-se que 74 (59,1\%) pais/responsáveis removeram o hábito de sucção de chupeta da criança de forma abrupta, e $10(12,5 \%)$ relataram que as crianças ficaram mais agitadas após pararem com o hábito. $\mathrm{O}$ hábito de sucção está relacionado a fatores emocionais; por essa razão, sua remoção deve ocorrer de forma gradativa pela possibilidade do desenvolvimento de hábitos ainda mais deletérios ou alterações no comportamento das crianças ${ }^{28}$.

A sucção além de satisfazer a necessidade nutritiva da criança, apresenta um aspecto emocional importante, fazendo com que a mesma muitas vezes recorra à chupeta, dedo ou artefatos para suprir suas necessidades emocionais ${ }^{25}$. Os pais/responsáveis ofertam a chupeta às crianças mesmo tendo o conhecimento da possibilidade de ocorrer maloclusões. Achado este fundamentado na associação estatisticamente significativa entre a oferta da chupeta à criança e o conhecimento sobre a relação entre a presença de hábi- 
tos não nutritivos e a ocorrência de maloclusão $(\mathrm{p}<0,0001$ e Qui-quadrado =60,123).

É lógico entender que não basta os profissionais de saúde solicitarem aos pais que a chupeta não seja usada. Deverão ser fornecidas orientações objetivando a conscientização sobre a importância do aleitamento materno não só para o não desenvolvimento dos hábitos de sucção não nutritiva ${ }^{29}$, mas também para a prevenção de doenças sistêmicas ${ }^{9,29-32}$. Outro aspecto importante é que segundo estudos ${ }^{1,6}$ a chupeta tem um papel importante na sucção, entretanto deve apresentar características que diminuam os efeitos maléficos na oclusão, como por exemplo ter o bico ortodôntico que melhor se ajusta ao palato.

Os pais devem considerar a origem do hábito de sucção, tentando diagnosticar, minimizar ou corrigir as causas de desconforto, medo e ansiedade da criança, pois quando o hábito perdura por um tempo prolongado, em muitos casos, existe falta de relacionamento materno-infantil. Com a idade, há a tendência natural de diminuir a frequência da prática do hábito, usualmente pela vergonha da ação. Na persistência, o hábito deverá ser gradativamente removido, com a ajuda de um psicólogo; e/ou um dentista que indique um aparelho ortodôntico, dificultando a criança de praticá- $10^{27}$.

A transmissão dessas informações, de forma clara e motivadora para a população, precisa ser realizada por profissionais da saúde de diferentes áreas de atuação, como médicos, cirurgiõesdentistas, enfermeiros, fonoaudiólogos e nutricionistas com o propósito de conscientizar os pais sobre as questões referentes à saúde bucal das crianças, principalmente quanto aos hábitos de sucção não nutritivos ${ }^{31}$. Idealmente, essas informações deveriam ser transmitidas às futuras mães durante as consultas do pré-natal ${ }^{33}$.

\section{Considerações finais}

Os dados do presente estudo revelam alta prevalência de crianças em idade pré-escolar que apresentam hábitos de sucção não nutritiva, destacando-se o uso da chupeta. Apesar da maioria dos pais saber que a sucção de chupeta poderia causar danos à saúde bucal, ofertavam-na a fim de acalmar e apaziguar a criança. Esses achados indicam a importância de valorizar e efetivar o trabalho multidisciplinar dos profissionais da saúde, em especial das equipes que atuam na atenção básica do Sistema Único de Saúde.

É necessária a criação e o desenvolvimento de políticas públicas consonantes com a realidade local e os valores socioculturais da população, notadamente na área da educação, a fim de estabelecer o reforço contínuo necessário para que novos conceitos sejam absorvidos, ocorram mudanças de comportamento e a formação de hábitos saudáveis. Dessa maneira, as ações educativas irão contribuir para o correto desenvolvimento do sistema estomatognático do pré-escolar e consequente integração social da criança dentro e fora da pré-escola e melhora da qualidade de vida desse grupo prioritário na atenção em saúde.

\section{Colaboradores}

CAS Garbin, RJ Martins, AJÍ Garbin, NP Souza, SAS Moimaz participaram igualmente de todas as etapas da elaboração do artigo.

\section{Agradecimentos}

À Coordenação de Aperfeiçoamento de Pessoal de Nível Superior pela bolsa de mestrado concedida. 


\section{Referências}

1. Sexton S, Natale R. Risks and benefits of pacifiers. Am Fam Physician 2009; 79(8):681-685.

2. Tenório MDH, Rocha JES, Fraga AB, Tenório DMH, Pereira PS. Sucção digital: observação em ultrasonografia e em recém-nascidos. Radiol Bras 2005; 38(6):435-438.

3. Barnett EM. Terapia oclusal en odontopediatría. Buenos Aires: Panamericana; 1978.

4. Turgeon-O'Brienn H, Lachapelle D, Gagnon PF, Laroque I, Maheu-Robert LF. Nutritive and nonnutritive sucking habits: a review. ASDC J Dent Child 1996; 63(5):321-327.

5. Canadian Paediatric Society. Recommendations for the use of pacifiers. Paediatr Child Health 2003; 8(8):515-519.

6. Bishara SE, Warren JJ, Broffitt B, Levy SM. Changes in the prevalence of nonnutritive sucking patterns in the first 8 years of life. Am J Orthod Dentofacial Orthop 2006; 130(1):31-36.

7. Lino AP. Hábitos e alterações da seqüência de erupção dentária. In: Lascala NT, organizador. Atualização clínica em Odontologia. São Paulo: Artes Médicas; 1982. p.31-35.

8. Moimaz SAS, Lolli LF, Garbin CAS, Saliba O, Saliba NA, Azevedo PS. Harmful oral suction habits in children: association with breastfeeding and family social profile. Rev Odonto Ciênc 2010; 25(4):355-360.

9. Moimaz SAS, Rocha NB, Garbin AJI, Saliba O. Relação entre aleitamento materno e hábitos de sucção não nutritivos. Cien Saude Colet 2011; 16(5):24772484.

10. Fófano CSN, Mialhe FL, Silva RP, Brum SC. Conhecimentos, atitudes e práticas maternas em relação ao uso da chupeta. Pesqui Bras Odontopediatria Clín Integr 2009; 9(1):119-123.

11. Sertório SCM, Silva IA. As faces simbólica e utilitária da chupeta na visão de mães. Rev Saude Publica 2005; 39(2):156-162.

12. Katz CRT, Rosemblatt A, Gondin PPC. Nonnutritive sucking habits in Brazilian children: effects on deciduous dentition and relationship with facial morphology. Am J Orthod Dentofacial Orthop 2004; 126(1):53-57.

13. Montaldo L, Montaldo P, Cuccaro P, Caramico N, Minervini G. Effects of feeding on non-nutritive sucking habits and implications on occlusion in mixed dentition. Int J Paediatr Dent 2011; 21(1):68-73.

14. Warren JJ, Bishara SE, Steinbock KL, Yonezu T, Nowak AJ. Effects of oral habits' duration on dental characteristics in the primary dentition. J Am Dent Assoc 2001; 132(12):1685-1693.

15. Graber TM. Etiologia da maloclusion, factores locales. In: Graber TM, organizador. Ortodoncia: teoria y pratica. $3^{a}$ Edição. Ciudad de México: Interamericana; 1974. p. 311-374.

16. Fonseca JS, Martins GA. Curso de estatística. 6a Edição. São Paulo: Atlas; 2001.

17. Ayres M, Ayres-Jr M, Ayres DL, Santos AAS. Bioestat 5.0. Belém: Sociedade Civil Mamirauá; 2007.

18. Bardin L. Análise do conteúdo. 3a Edição. Lisboa: Edições 70; 2004.
19. Brasil. Ministério da Saúde (MS). Pesquisa Nacional de Saúde Bucal: 2010: nota para a imprensa. [página na Internet]. [acessado 2011 abr 4]. Disponível em: dab.saude.gov.br/cnsb/sbbrasil/SBBrasil 2010_Nota_Imprensa.pdf

20. Aznar T, Galán AF, Marin I, Domínguez A. Dental arch diameters and relationships to oral habits. Angle Orthod 2006; 76(3):441-445.

21. Bittencourt LP, Modesto A, Bastos EPS. Influência do aleitamento sobre a frequência dos hábitos de sucção. Rev Bras Odontol 2001; 58(3):191-193.

22. Tomita NE, Bijella VT, Franco LJ. Relação entre hábitos bucais e má-oclusão em pré-escolares. Rev Saude Publica 2000; 34(3):299-303.

23. Cunha SRT, Corrêa MSNP, Oliveira PML, Schalka MMS. Hábitos bucais. In Corrêa MSNP, organizador. Odontopediatria na primeira infância. São Paulo: Ed. Santos; 1998. p. 561-576.

24. Christensen J, Fields H. Hábitos bucais. In: Pinkham $\mathrm{JR}$, organizador. Odontopediatria da infância à adolescência. 2a Edição. São Paulo: Artes Médicas; 1996. p. $400-407$.

25. Martins RJ, Garbin AJI, Garbin CAS, Moimaz SAS. Chupeta: amiga ou inimiga? Rev Assoc Paul Cir Dent 2003; 57(1):32-35.

26. Instituto Nacional de Metrologia, Qualidade e Tecnologia (Inmetro). Informação ao consumidor: chupetas. [página na Internet] 2009. [acessado 2012 jan 17]. Disponível em: www.inmetro.gov.br/consumidor/ produtos/chupetas.asp

27. American Dental Association. Thumb sucking and pacifier use. J Am Dent Assoc 2007; 138(8):1176.

28. Walter LRF, Ferelle A, Issao M. Odontologia para o bebê: odontopediatria do nascimento aos 3 anos. São Paulo: Artes Médicas; 1996.

29. Albuquerque SLL, Duarte RC, Cavalcanti AL, Beltrão EM. A influência do padrão de aleitamento no desenvolvimento de hábitos de sucção não nutritivos na primeira infância. Cien Saúde Colet 2010, 15(2):371-378

30. Corrêa MSNP. Odontopediatria na primeira infância. 2a Edição. São Paulo: Ed. Santos; 2005.

31. Antunes LS, Antunes LAA, Corvino MPF, Maia LC. Amamentação natural como fonte de prevenção em saúde. Cien Saude Colet 2008, 13(1):103-109.

32. Moura LFAD, Moura MS, Toledo OA. Conhecimentos e práticas em saúde bucal de mães que frequentaram um programa odontológico de atenção materno-infantil. Cien Saude Colet 2007, 12(4): 1079-1086.

33. Martins RJ, Garbin CAS, Garbin AJI, Moimaz SAS. Sucção não-nutritiva:importância da integração entre pediatria e odontologia. Rev Assoc Paul Cir Dent 2005; 59(6):443-447.

Artigo apresentado em 30/11/2012

Aprovado em 23/03/2013

Versão final apresentada em 01/04/2013 